\title{
Service for Good
}

\section{Grundlagen und Methoden zum Design personennaher Dienstleistungen}

\author{
Susanne Robra-Bissantz' ${ }^{1}$ Anna M. Lux ${ }^{1}$ Christoph Lattemann²
}

Angenommen: 15. Juni 2021 / Online publiziert: 4. August 2021

(c) Der/die Autor(en) 2021

\section{Zusammenfassung}

Vorspann: Die digitale Transformation geht Hand in Hand mit einem radikalen Wandel der Märkte. Gewinner beider Entwicklungen sind solche Unternehmen, die das Wohlergehen von Menschen in den Vordergrund stellen und „Service for Good" anbieten.

Auf dem Weg zu Service for Good: Die Entwicklungen der vergangenen Jahre führen uns vor Augen, dass sich grundlegende Annahmen über Märkte, Produkte und Wertschöpfung verändern. Die Gewinner der digitalen Transformation machen vor, was theoretische Fundamente der modernen Betriebswirtschaftslehre erklären: Die Zukunft liegt in der Gestaltung von Services, die auf die persönliche Situation von Menschen ausgerichtet sind. Eine Herausforderung des Human Life Engineering ist es, Potenziale der Digitalisierung für personennahe Dienstleistungen nutzbar zu machen. Der vorliegende Beitrag beleuchtet hierfür notwendige Grundlagen der Service Logik und stellt mit der Service Canvas einen anwendungsorientierten Gestaltungsrahmen vor. Hieraus leitet sich ein Methodenset zum Design personennaher Dienstleistungen ab, das im Rahmen eines Forschungsprojekts eine erste praktische Anwendung fand. Ziel ist es, Unternehmen in der Gestaltung zukunftsorientierter Angebote zu begleiten, die das Wohlergehen von Menschen in den Vordergrund stellen: Service for Good.

\section{Digitalisierung und Dienstleistungen}

Sind wir doch einmal ganz ehrlich. Es ist die Digitalisierung, die von Unternehmen fordert, sich darauf zu besinnen, was ihre eigentliche Aufgabe ist: Menschen darin zu unterstützen, dass sie ihr Leben so leben können, wie sie wollen [1]. Diejenige Digitalisierung, die gleichzeitig den Ruf hat, Jugendliche ihrer sozialen Beziehungen zu berauben und

Susanne Robra-Bissantz

s.robra-bissantz@tu-braunschweig.de

Anna M. Lux

anna.lux@tu-braunschweig.de

Christoph Lattemann

c.lattemann@jacobs-university.de

1 Lehrstuhl für Informationsmanagement, Institut für Wirtschaftsinformatik, Technische Universität Braunschweig, Braunschweig, Deutschland

2 Professor for Business Administration and Information Management, Jacobs University Bremen, Bremen, Deutschland
Märkte vom gemütlichen Einzelhandel aus der Stadt in das unpersönliche, ja gefährliche digitale Netz zu verschieben.

Denn bei näherer Betrachtung zeichnet alle derzeitigen Gewinner der digitalen Transformation ein wesentliches Merkmal aus: Ihr Angebot ist genau darauf ausgerichtet, dem Menschen einen besonderen Wert zu versprechen [2]. Den Wert, den er zur Verbesserung seiner Lebenssituation sucht. Sei es in der einfachen Information, Beratung, im Vergleich und der Empfehlung von Produkten, aber auch unabhängig von einem Produkt, das er kauft. Unternehmen versprechen ihren Kunden Dienstleistungen, die gesamte Problemstellungen, wie beispielsweise in der Mobilität, lösen. Wir beobachten Phänomene des Teilens, Tauschens und des Abonnements von Gütern, die Menschen, statt sie nutzlos zu besitzen, lieber besitzlos nutzen [3]. Ebenso nehmen wir wahr, dass ein Angebot für einen Kunden nicht von einem institutionalisierten Anbieter wie einem Unternehmen kommen muss. Stattdessen entscheiden Kunden zunehmend, eigene Kompetenzen auszuprägen und diese für sich im Do-it-yourself oder für andere, als Maker (z.B. bei Uber oder Airbnb), zu nutzen. 
Grundlegende Paradigmen über Märkte und deren Beziehung zum Leben ihrer (privaten) Kunden stehen, offensichtlich in Zeiten der Digitalisierung, auf dem Prüfstand [4]: Ist es wirklich das Produkt, an dem Unternehmen ihre Innovationstätigkeit ausrichten müssen oder vielleicht der Wert, den ein Kunde sich davon erwünscht? Ist der Kunde dann eine Zielgruppe oder vielmehr ein Partner, der aktiv entscheidet, welcher Anbieter ihn in seinem Leben unterstützt?

Eine Antwort aus der (im Entstehen befindlichen) Community Service for Good ist es, private Personen auf digitalen Märkten als die aktiven Partner zu sehen, die darüber entscheiden, welche Angebote sie nutzen, um für sich einen Wert zu schaffen und damit ihre Lebenssituation zu verbessern. Ein Ansatz des Human Life Engineering, aus dem Blickwinkel der Wirtschaftsinformatik, besteht dann darin, die Potenziale der Digitalisierung zum Design von marktlichen Angeboten zu nutzen, die genau auf die persönliche Lebenssituation von Menschen ausgerichtet sind: Wir nennen sie personennahe Dienstleistungen. Gleichzeitig hilft die Sichtweise von Service-for-Good-Unternehmen, die in der digitalen Transformation Fuß fassen oder verbleiben wollen.

Design (oder Engineering) erfordert Methoden. Diese basieren auf den im folgenden Kapitel dargestellten Grundlagen von Service for Good und führen zu einer Service Canvas: Ein Rahmen für Designmethoden personennaher Dienstleistungen. Abschließend zeigen wir die beispielhafte Anwendung einiger Methoden im praktischen Kontext unseres Metaprojekts BeDien der Förderlinie „Personennahe Dienstleistungen“ des BMBF (Bundesministerium für Bildung und Forschung).

\section{Service for Good}

Die Bezeichnung Service for Good steht zum einen dafür, dass es, unserer Meinung nach, die wesentliche Chance für
Unternehmen auf digitalen Märkten ist, in ihren Aktivitäten danach zu streben, dass letztendlich personennahe Dienstleistungen für private Kunden angeboten werden. Zum anderen trägt die Bezeichnung ,for Good“ auch in sich, dass das Wohlergehen von Menschen im Zentrum des Interesses steht.

Grundlage ist die Service Logik aus der Betriebswirtschaftslehre [5]. Sie sieht die Aufgabe von Akteuren auf Märkten letztlich darin, Services anzubieten, die dem privaten Kunden in seinem Leben wertvoll sind. Die Digitalisierung auf Märkten macht Services im Sinne der Service Logik möglich. Gleichzeitig bietet sie die Chance, unternehmerische Angebote als personennahe Dienstleistungen zu gestalten. Die personennahe Dienstleistung grenzt sich von der eher geläufigen produktnahen dadurch ab, dass sie nicht, z.B. als Wartungsleistung, an einem Produkt ausgerichtet ist. Sie richtet sich vielmehr danach, was Menschen ihr Leben verbessert. „Personennah“ ist dem „,humanzentrierten“ Ansatz der Informatik sehr ähnlich: Nur interessieren statt der menschlichen Eigenschaften vielmehr persönliche Bedürfnisse und Präferenzen.

\section{Service Logik}

Die Service Logik [6] oder auch servicedominierte Logik [7] geht davon aus, dass jegliche Geschäftstätigkeit eine Dienstleistung ist. Damit wird die, schon seit Langem schwierige, Trennung zwischen Sachgut und Dienstleistung aufgehoben: In jedem Fall nutzt ein Anbieter seine Kompetenzen dazu, einem betrieblichen oder privaten Kunden einen Service zu bieten, der dazu dient, dass dieser seine Lebenssituation verbessert. Hierdurch werden 2 Grundpfeiler des wirtschaftlichen Handelns wesentlich verändert: die Rolle des Produktes und die Rolle des Kunden (vgl. Abb. 1).

Das Produkt ist in seinem Selbst nicht mehr wertvoll [8]. Die Service Logik argumentiert, dass es nicht der Kauf eines Sachguts ist, der Bedürfnisse von Kunden deckt. Stattdessen sind es die Kompetenzen, beispielsweise zur Pro-

Abb. 1 Service Logik

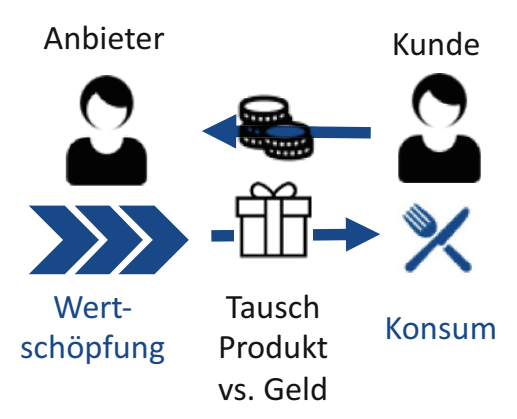

Traditionelle Sicht vs. Service Logik

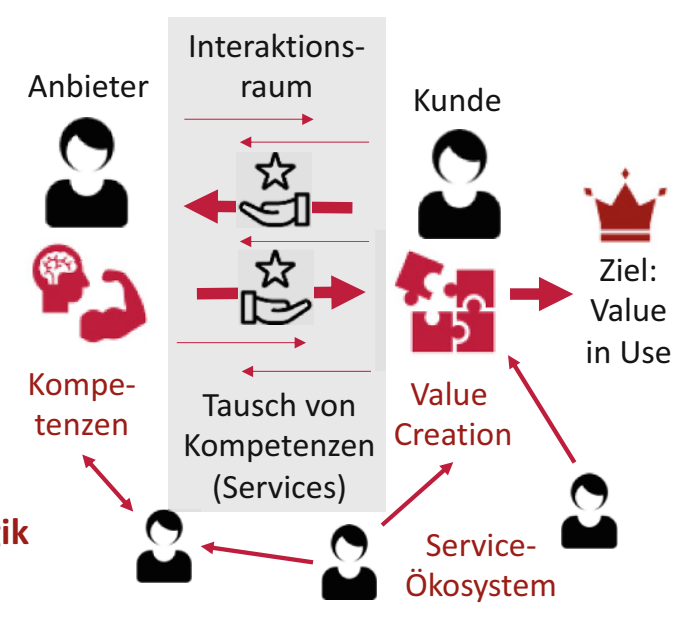


duktion des Produkts oder in der Dienstleistung, über die der Kunde nicht selber verfügt und die er vom Anbieter in Anspruch nimmt, um sich selber einen Wert (Value in Use) zu schaffen. So versprechen Kompetenzen, wie z.B. ein Auto zu bauen oder Busfahrten zu organisieren, jeweils den Wert der Mobilität während ein Haarschnitt des Friseurs zum Value in Use des besseren Aussehens oder des gesünderen Haars führt. Immer stellt sich dieser Nutzwert als rein individuell und kontextbezogen dar $[6,9,10]$.

Der Kunde und seine eigene Wertschaffung rücken in den Mittelpunkt der Serviceorientierung. Mehr über den Kunden und seine Bedürfnisse zu wissen, von ihm eingeladen zu werden zu seinem Wert beizutragen und ihn in unternehmerische Prozesse zur Entwicklung passender Angebote zu integrieren, wird ausschlaggebend für den Erfolg am Markt [8]. Zudem entscheidet der Kunde stets selbst und aktiv, mit wem er zusammenarbeiten möchte, welche Unternehmenskompetenzen als Service zur Co-Creation seines Werts beitragen können und wie und wann er sich selber einbringt [6]. Um als Co-Creator zu überzeugen, braucht es, als neue Währungen neben dem Geld, Kompetenzen wie Wissen über den Kunden oder Reputation und zudem einen Interaktionsraum zwischen den Partnern [7]. Gleich, ob dieser Interaktionsraum virtuell oder physisch ist, proaktiv vom Unternehmen aufgebaut wird, sich durch Suchen des Kunden eröffnet oder aufgrund von Vermittlung Dritter - die Gestaltung der Interaktion selbst spielt in der gemeinsamen Wertschaffung eine entscheidende Rolle. Jede Interaktion ist wertvoll, denn gut gestaltete Kontaktpunkte mit dem Kunden bieten einen nicht zu vernachlässigenden, eigenen Wert - den Value in Interaction [11]. Dieser entsteht beispielsweise bereits vor dem Kauf durch eine gut gelungene Inspiration des Kunden, während dieser noch überlegt, wie er ein Problem lösen könnte, anschließend dadurch, dass gemeinsam eine möglichst gut zum Problem passende Dienstleistung - beispielsweise über Beratung oder Konfiguration - gefunden wird, sowie nach dem Kauf, indem der Anbieter den Kunden darin unterstützt, möglichst lange und wirkungsvoll Nutzen aus Produkt oder Dienstleistung zu ziehen.

In der Service Logik ist jeder Akteur zugleich derjenige, der seinen eigenen Wert schafft und zum Wert anderer beiträgt. Dabei ist der Value in Use so definiert, dass er ganze Lebenssituationen verbessert. Hierzu sind eine Vielzahl miteinander verwobene Akteure notwendig: Es bilden sich Service-Ökosysteme [12].

\section{Die Rolle der Digitalisierung}

Digitalisierung und der Wandel zu einer serviceorientierten Geschäftstätigkeit (Servicification) werden als 2 Seiten derselben Münze gesehen [13]. Digitalisierung ermöglicht bessere Dienstleistungen - und genau dann liegt es an der
Digitalisierung, auch Anstöße für personennahe Dienstleistungen zu geben. Allerdings verlangt eine derartige Sicht, einen stark vereinfachten, abstrahierenden Blick auf die Digitalisierung zu werfen.

Mit dem Megatrend der Konnektivität zeigt das $\mathrm{Zu}$ kunftsinstitut auf, dass Digitalisierung nicht allein neue Technologien betrifft, sondern gleichzeitig einen sozialen und kulturellen Wandel herbeiführt [23]. In der heute digital geprägten Realität des wirtschaftlichen Handelns ist damit die Vernetzung, die jeglichen Privaten geradezu gleichberechtigt in die Kommunikation mit betrieblichen Partnern einbindet, die wesentliche Grundlage einer Servicification. Denn genau über digitale Netze wird der Kunde selbst aktiv. Er wird nicht mehr als passive Zielgruppe angesprochen und von etwas überzeugt, sondern wählt das am besten zu seiner Lebenssituation passende Angebot aus. Und nicht nur das: Jegliche Website oder App eines Unternehmens wird durch vernetzte digitale Services zum 24/7 verfügbaren Ansprechpartner und bietet Value in Interaction: ausführliche Information und Beratung, Individualisierung oder Konfiguration sowie an den Kauf anschließende Services [8, 11].

Mit der Ubiquität von Informationssystemen sind Unternehmen und Kunden potenziell durchgehend in digitalen Interaktionsräumen verbunden [11]. Damit bleibt der Kanal zum Kunden offen und Anbieter stehen immer wieder für gesamte Problemlösungen von Kunden zur Verfügung. Digitale Sensorik, bereits in der Ortserkennung, trägt hier ohne weitere Interaktion des Kunden dazu bei, Aufschluss über dessen Situation und potenzielle Bedarfe zu erhalten. Hierfür steht auch entsprechendes Wissen, über Clouds, überall und zusammengeführt zur Verfügung. Eigenständige Software- bis hin zu Hardwarekomponenten können z. B. unterstützt durch künstliche Intelligenz darauf mit entsprechenden (digitalen) Services reagieren und machen umfangreiche, persönliche Dienstleistungen, wie z.B. die Begleitung in Lern- oder Mobilitätsprozessen, durch digitale Assistenten effizient und effektiv möglich [8].

Die Nutzung der allseits verfügbaren Vernetzung zur multimedialen oder sozialen Kommunikation befördert dezentrales Handeln und kollaborative Interaktionen. In Service-Ökosystemen aus vielen Partnern, die auch Private mit ihren dezentralen Dienstleistungsangeboten einschlieBen, entstehen Peer-to-Peer-Angebote, z.B. zum Tauschen und Teilen. Emotionale und soziale digitale Interaktionen, z.B. mithilfe der Ansätze sozialer Medien, ermöglichen eine Zusammenarbeit aller Partner, ob betrieblich oder privat, die auf Verbundenheit und Vertrauen basiert. Ins Zentrum des Interesses rückt der Anspruch, das eigene Angebot in Zusammenarbeit mit seinem Nutzer immer besser an diesen anzupassen [14]. Je besser es dabei gelingt, Vertrauen in Beziehungen zu schaffen, desto mehr werden die Partner 
bereit sein, ihre Daten beizutragen und aktiv am Netzwerk teilzunehmen [15].

\section{Die Service Canvas als Rahmen zum Design personennaher Dienstleistungen}

Als Teilergebnis des Metavorhabens „BeDien“ [16] zur BMBF-Förderlinie „Personennahe Dienstleistungen“, ist auf Basis von Service Logik und Digitalisierung die Service Canvas entstanden [17]. Sie stellt die wesentlichen Gestaltungsbereiche für Unternehmen aus einer Dienstleistungsperspektive den wesentlichen Entwicklungslinien in der digitalen Transformation gegenüber und spannt so einen Rahmen für die Konzeption und Umsetzung der personennahen Dienstleistungen aus den 8 Förderprojekten. Damit bildete die Service Canvas von Beginn des Metavorhabens an eine strukturierte Grundlage für Diskussionen, Austausch und gegenseitige Wissensvermittlung unter den einzelnen Projekten sowie mit dem BeDien-Team. Im Projektverlauf wurde die Service Canvas kontinuierlich an neue Erkenntnisse, auch aus den Projekten, angepasst.

Die Service Canvas umfasst heute 3 aus der Service Logik und eigener Forschung entwickelte Gestaltungsbereiche: Value in Use, Value in Interaction sowie ServiceÖkosysteme (vgl. Abb. 2). Der Value in Use ist darauf ausgerichtet, genau auf den Wert zu achten, den sich der einzelne Mensch oder Akteur in seiner Situation vom Angebot des Unternehmens wünscht. Im Value in Interaction ist die Relevanz von Interaktionen in Prozessen und Beziehungen zwischen Akteuren abgebildet. Das Service-Ökosystem weitet den Blick vom einzelnen Unternehmen auf das Netzwerk, das alle, beispielsweise auf einer digitalen Plattform kooperierenden, Akteure umfasst.

Als wesentliche Entwicklungslinien in der digitalen Transformation zeigt die Service Canvas in ihrer ersten Spalte die Adaption auf und damit die Anpassung des
Serviceangebots an den Kunden und seine Bedürfnisse, sowohl, was den Value in Use als auch den Value in Interaction betrifft: der passende, individuelle Service, gelungene Customer Journeys über Inspiration und Matching bis hin zur Problemlösung, auch über mehrere Akteure und ggf. deren potenziellen Value in Use.

Die Integration, in der zweiten Spalte, beschreibt ein wesentliches und durch Digitalisierung begünstigtes Charakteristikum der Co-Creation in der Service Logik: jeder Akteur in der Anbieter-Kundenbeziehung, aber auch im ServiceÖkosystem, bringt besondere Kompetenzen in die gegenseitige Problemlösung ein. Aus Sicht der Akteure gilt es zu überlegen, ob für jeden die erhaltenen und geleisteten Services ausbalanciert sind und ob ggf. zusätzliche Partner Lücken im Service-Ökosystem schließen können. Aus Sicht der Kompetenzen muss abgeschätzt werden, ob ein Service für einen Partner einen Wert darstellt oder er die Kompetenz, z. B. im Sinne des Do-it-yourself, lieber selbst beiträgt. Unter Umständen ungern lässt ein Akteur dagegen wertvolle Kompetenzen, wie beispielsweise Wissen über seine eigenen Prozesse oder seine Reputation, einfließen. Hier spielt es für eine Reihe von möglichen Geschäftsmodellen eine Rolle, inwieweit die in der nächsten Spalte angesprochene Kollaboration zwischen den Partnern ausgeprägt ist. Sie zeigt Potenziale dazu auf, Beziehungen gegenseitig, mit gemeinsamen Zielen, auf Basis von Verbundenheit und Vertrauen zu gestalten. Letztlich stellt sich die Frage nach einem digitalen Design - der Dienstleistung, der Beziehungen zwischen den Partnern sowie des gesamten ServiceÖkosystems. Diese Entwicklungslinie ist mit Bedacht in die letzte Spalte der Service Canvas gesetzt, da sich der Grad der Digitalisierung in einem postdigitalen Sinne nicht an den Potenzialen der Technologie, sondern an ihrem maßgenauen Einsatz zur Verbesserung der Lebenssituation des Einzelnen ausrichtet.

Das BeDien-Team nutzt die Service Canvas sowie ihre Spalten, Zeilen und Felder auch zur Sammlung von Desi-

Abb. 2 Die Service Canvas

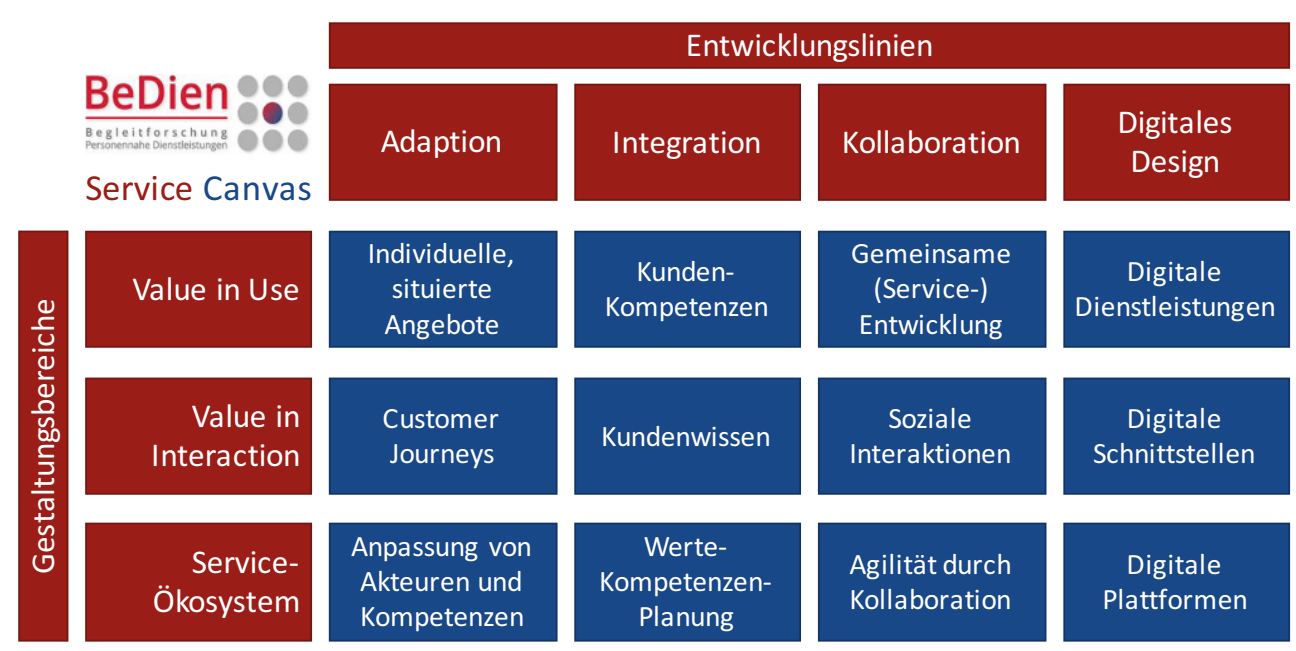


gnmethoden, die potenziell jegliches (traditionelle) Unternehmen dazu anregt, sein häufig produktorientiertes Angebot als personennahe Dienstleistung in digitalisierte Märkte zu transformieren: das Service-for-Good-Methodenset.

\section{Das Service-for-Good-Methodenset im Praxistest}

Die Projekte der Förderlinie stammen aus unterschiedlichen Anwendungsbereichen, wie z. B. Wohnen, Nachbarschaftshilfe oder Bildung. Gemein ist ihnen, dass sie mit einer häufig aus der technologischen Forschung stammenden Projektidee für eine digital unterstützte personennahe Dienstleistung starteten. Diese soll im Laufe der Förderdauer zum einen konzipiert und mit betrieblichen Partnern umgesetzt und zum anderen auch in einen Vorschlag münden, wie sie nachhaltig im betrieblichen Umfeld eingeführt und erfolgreich sein kann.

Nahe liegen hier Methoden des Design Thinking sowie der Geschäftsmodellentwicklung. Beide fließen in das Service-for-Good-Methodenset ein. Aus der Geschäftsmodellentwicklung entspringt die Berücksichtigung verschiedener Gestaltungsbereiche und Partner: hier die Gestaltungsbereiche der Service Canvas mit den Akteuren eines ServiceÖkosystems. Das Design Thinking trägt Voraussetzungen der kreativen Ideenentwicklung bei: Die Anwendung der Methoden erfolgt auch für Service for Good in Teams, die schrittweise an einem Shared Mental Model arbeiten. Hierzu starten die BeDien-Workshops jeweils mit Impulsvorträgen zur Service Canvas und verpacken die Erkenntnisse in einprägsame Merksätze. Auch die aus dem Denken des Designers abgeleiteten Techniken des Divergierens und Konvergierens sowie Induktion, Deduktion und Abduktion sind aus dem Design Thinking übernommen. Jedoch richtet sich das Service-for-Good-Methodenset spezifischer an der Theorie der Service Logik aus und nutzt zur Abduktion gezielt Kartensets mit Patterns aus der Praxis der personennahen Dienstleistung, die in den letzten Jahren mit

Abb. 3 Designmethoden für den Value in Use
Studierenden der Wirtschaftsinformatik an der TU Braunschweig zusammengetragen wurden, zur Inspiration für die Weiterentwicklung des eigenen Geschäfts.

In BeDien entstehen damit (unter anderem) Designmethoden für personennahe Dienstleistungen. Diese Gestaltungsleistung ist Teil des dazugehörigen Forschungsprojekts, das, angelehnt an das Action Design Research, jeweils Interventionen mit dem Service-for-Good-Methodenset im Umfeld der Stakeholder - hier der Projekte - gestaltet und mit diesen evaluiert. Gleich einem Design-Science-Projekt ist das letztliche Ziel dieses Forschungsprojekts die Gestaltung von Designmethoden als Artefakte, die, abstrahiert, von weiteren Forschern in diesem Bereich weiterentwickelt und evaluiert werden sowie in der Praxis als Methodenset für Gestalter von personennahen Dienstleistungen im Sinne des Service for Good zur Verfügung stehen. Vorliegender Beitrag stellt in diesem Kontext einen Erfahrungsbericht aus den ersten Iterationen dieses Methodensets dar.

\section{Design Challenge: Value in Use}

Das Denken an einen Value in Use einer Dienstleistung will geübt sein. Im Design-Workshop zeigt hierzu ein Impulsvortrag auf, dass unter Umständen verschiedene, ganz unterschiedliche Akteure miteinander konkurrieren, wenn der Kunde beispielsweise anstrebt, mit seiner Kleidung einen gepflegten Eindruck zu machen: der Anbieter von Bügeleisen oder derjenige von Steamern, aber auch der Bügelservice oder der Hersteller von bügelfreien Kleidungsstücken. Es gilt damit der Merksatz Sein ist besser als haben. Kunden suchen Werte, nicht Produkte. Jedes Produkt und jede Dienstleistung kann für einen Kunden unterschiedliche Werte bieten und umgekehrt führen häufig unterschiedliche unternehmerische Angebote zum gleichen Value in Use.

Für die Förderprojekte gilt es zunächst herauszuarbeiten, inwiefern ihre geplante Dienstleistung die Lebenssituation von Menschen verbessern kann. Dazu dienen Methoden wie die Tütenchallenge, die Schlagzeilenmethode oder die Methode From Service to Value (vgl. Abb. 3). Bereits einge-

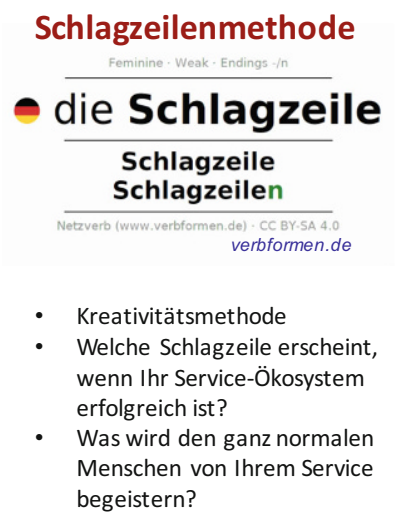

Tütenchallenge

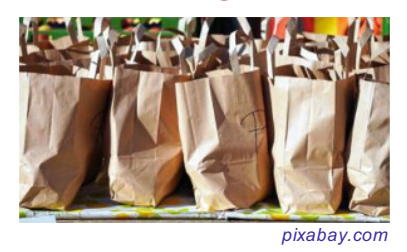

- Kreativitätsmethode

- Welche Argumente findet ein guter Verkäufer für eine Tüte?

- Wofür kann man eine Tüte gebrauchen?

- Anschließend: nicht Tüte sondern Ihre Dienstleistung.
From Service to Value

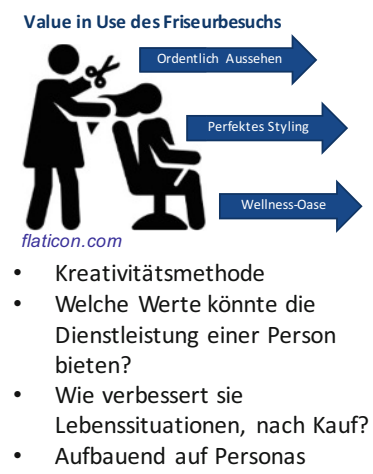


führte Methoden, wie Personas oder User Stories, geben zudem Aufschluss über individuell unterschiedliche Werte, wie am Beispiel des Projekts ProDruck erkennbar wird. Hier kann ein selbst gestaltetes 3D-gedrucktes Gadget einer Person dazu dienen, mehr Unabhängigkeit im Alltag zu erlangen, während es für eine andere Person den Wert einer kreativen Eigenleistung hat.

Häufig erkennen die Projekte, während der Moderation der Methode From Service to Value durch Teammitglieder von BeDien, dass sich der eigentliche Wert (Value in Use) des Kunden ihrer Dienstleistung auf vorher nicht berücksichtigte, übergeordnete Lebenssituationen bezieht. Dazu fragen BeDien-Mitarbeiter zunächst nach dem Nutzen der zu entwickelnden Dienstleistung. Hier wurde im Beispiel des Projekts $A R B A Y$ ganz richtig die Darstellung von Möbelstücken mithilfe von Augmented Reality (AR) in der eigenen Wohnung genannt. Doch eine AR-Darstellung von Möbeln allein löst kein Kundenproblem. Gezieltes Nachfragen führte zunächst zum Value in Use einer gezielten Möbelberatung bis dahin, dass es dem Kunden schlussendlich um ein möglichst seinen Wünschen entsprechendes Wohnen geht. Dies wiederum eröffnet Ideen für weitere Partner, neben dem Möbelhaus beispielsweise Innenarchitekten, Tischler oder Elektrotechniker.

Ähnlich adressierte das Projekt Athene 4.0 zum Start der Methode From Service to Value die Unterstützung des Handwerks in seiner täglichen Arbeit. Jedoch ist auch dies kein Selbstzweck und es prägten sich letztlich verschiedene Werte beim privaten Kunden aus. So bietet die geplante digitale Plattform für Handwerker dem Kunden einen Wert darin, dass er seine Aufträge an Handwerker transparent nachverfolgen kann. Und weitergehend verbessert Athene 4.0 die Lebenssituation der Kunden: Sie können, auch wenn Reparaturen notwendig werden, ein sorgenfreies Leben in ihrer Wohnung oder ihrem Haus führen.

Ein von BeDien entwickeltes Kartenset regt an, darüber nachzudenken, ob der gewünschte Value in Use vielleicht (vom Konkurrenten) anders oder besser angeboten werden kann. Es trägt die Bezeichnung Value meets (digital) Service. Das Projekt Living Smart beispielsweise entwickelt weitere, wohnungsnahe Dienstleistungen, auf Basis des von ihnen identifizierten Werts, rundum versorgt in der eigenen Wohnung zu leben. Zusätzlich können Projekte mit der Methode Society Radar überprüfen, ob angestrebte Werte sich aufgrund aktueller gesellschaftlicher Trends ebenfalls verändern, wie z.B. in der Pandemie.

Als sehr hilfreich im Einstieg hat sich die Methode Mein Service - Dein Service erwiesen, in der ein Mitarbeiter eines Projekts dem Mitarbeiter eines anderen Projekts seine Dienstleistung erklärt und letzterer diese dann einer größeren Gruppe in eigenen Worten vorstellt. Innerhalb dieses Vorgehens wird sehr schnell deutlich, was der Kern der Dienstleistung für den Kunden sein kann. Ähnlich ging es in einem weiteren Workshop von BeDien darum, mithilfe der Methode Elevator Pitch die Erkenntnisse der Verbundprojekte für die Öffentlichkeitsarbeit trotz ihrer Komplexität zielgerichtet und prägnant zu kommunizieren.

\section{Design Challenge: Value in Interaction}

Die Berücksichtigung des Value in Interaction startet in Design-Workshops mit der Diskussion, ob ganz grundsätzlich der persönlichen, auch emotionalen Interaktion $\mathrm{zu}$ wenig Wert zugewiesen wird: „Erst wenn der letzte Einzelhändler in der Innenstadt geschlossen hat, erst wenn wir unsere Frühstücksbrötchen bei Amazon bestellen und erst, wenn eines Morgens der Pflegeroboter an unser Bett tritt, uns bettet und fragt, wie unsere Nacht war - erst dann werden wir erkennen, dass persönliche Interaktion wertvoll ist" [18]. Der gemeinsame Merksatz lautet: Worte zählen genauso viel wie Taten. Interaktionen sind wertvoll.

Daran schließen sich Designmethoden an, die zunächst dediziert die Customer Journey, aus dem Blickwinkel des Kunden, betrachten und die gemeinsame Beziehung über Interaktionsräume stärken, die den Kunden sowohl vor als auch nach der Dienstleistung begleiten: mit Kartensets $\mathrm{zu}$ Pre-Service- und Post-Service-Patterns. Diese, meist digitalen, Services dienen dazu, Kunden nicht zu verlieren, indem sie diese bereits vor Kauf durch die Phasen der Inspiration oder des Matching leiten. Nach der Dienstleistungserbringung werden die Kunden durch Dienste zur Werterweiterung, oder -erhaltung weiterhin begleitet und so lange nicht alleine gelassen, bis eine neue Dienstleistung fast natürlich beim selben Anbieter erfragt wird.

Ein anderer Aspekt des Value in Interaction besteht darin, wertvolle Kompetenzen der Partner für das eigene Geschäftsmodell zu gewinnen. Mit den Collaboration-Patterns wird, ebenfalls über ein Kartenset, geprüft, ob Potenziale dazu bestehen, die Beziehung, z. B. zu Kunden, kollaborativer zu gestalten. Dazu gehört beispielsweise, sie transparent in Innovationen einzubeziehen oder ihnen die Möglichkeit für Feedback zu geben. Wertvolle Services werden erst dann möglich, wenn Kunden auf der Basis von Verbundenheit und Vertrauen im Rahmen von Interaktionen Wissen über ihre Bedarfe zur Verfügung stellen. Für alle Partner gilt der Merksatz: Kontrolle ist gut, Vertrauen ist besser.

In der Anwendung der genannten Methoden, die auf Kartensets mit Patterns beruhen (vgl. Abb. 4), wird durch Mitglieder des BeDien-Teams jeweils eine Karte gezogen, das entsprechende, aus Best-Practice-Beispielen gewonnene Muster erläutert und dann mit den Projektmitgliedern diskutiert, ob sie in ihrem Projekt beispielsweise die Möglichkeit der Inspiration, der besseren Werterhaltung oder der besseren Zusammenarbeit mit oder unter Kunden sehen.

Das Projekt Lida erarbeitete beispielsweise als Kern seiner Plattform zur betrieblichen Weiterbildung einen Kreis- 


\section{Patterns: Kartensets zum Design im Bereich Value in Interaction (Beispielkarten)}
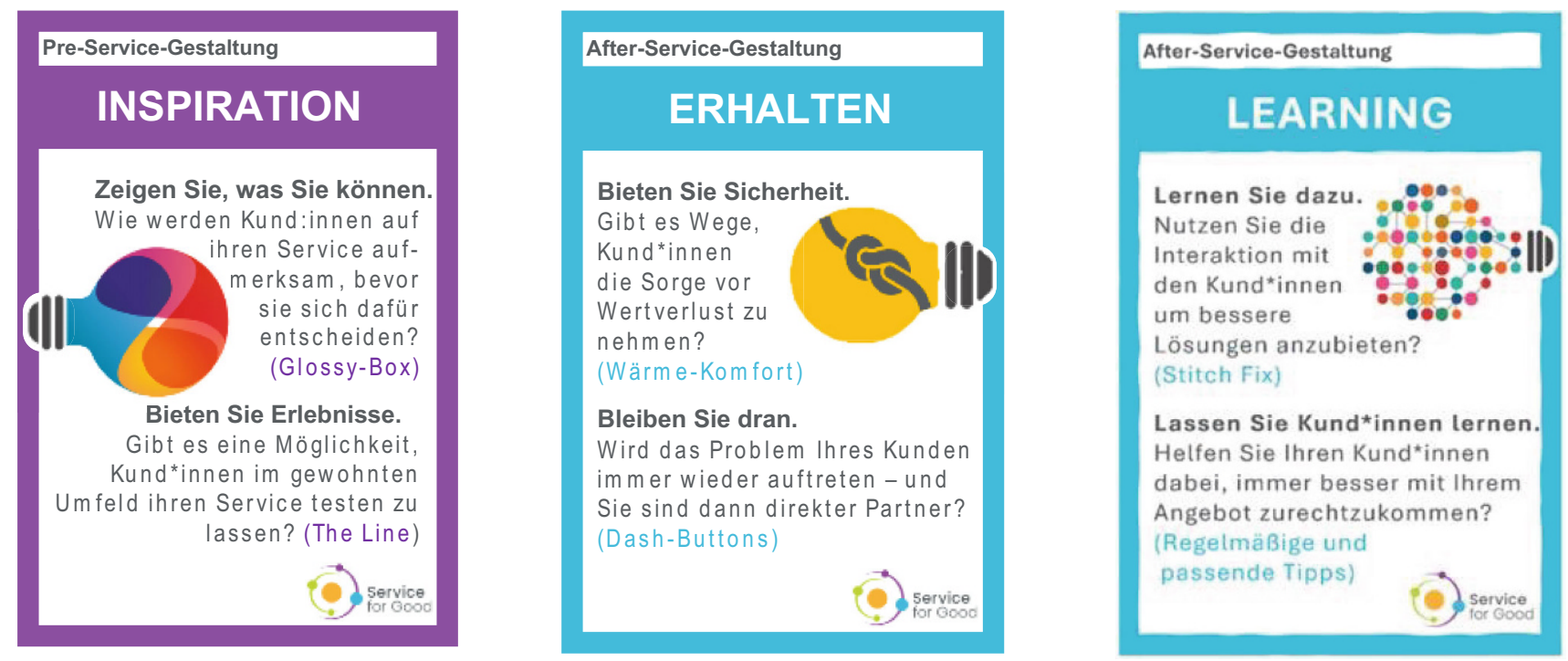

Abb. 4 Designmethoden (Patterns) für den Value in Interaction

Abb. 5 Basismethode im Design von Service-Ökosystemen

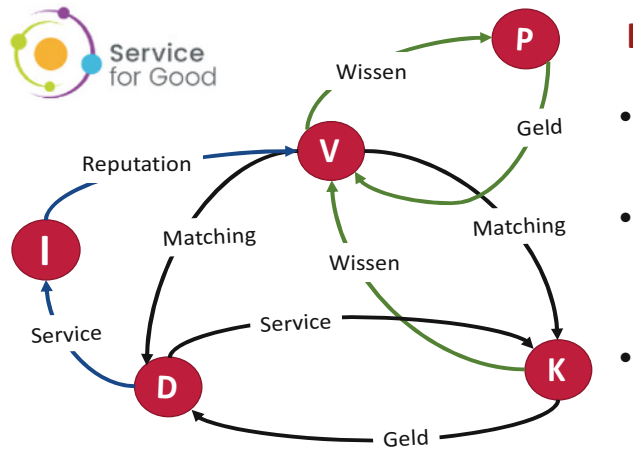

\section{Design ES (Ecosystems) wertorientiert}

- Intuitive, visuelle Gestaltung des gesamten Service-Ökosystems

- Iterative Erweiterung einer BasisDienstleistung oder Basis-Plattform um weitere e-Services und wichtige Akteure

- Jeweils: Check der Erfolgschancen und Austauschbeziehungen lauf aus Feedback der Lernenden und einer entsprechenden Anpassung der Dienstleistung - um diese stetig zu verbessern. In den Projekten INSELpro und KUSTOMA ist Vertrauen der Schlüssel zur Zusammenarbeit von Anbietern haushaltsnaher Dienstleistungen bzw. aus dem Bereich der Kinderbetreuung, deren potenziellen Nutzern sowie den jeweils vorgesehenen Vermittlerrollen. Basierend auf Impulsen und Diskussionen aus BeDien-Workshops, zeigen 3 Veröffentlichungen auf (vgl. [19-21]), wie mithilfe von digitalen Diensten der Aufbau von Vertrauen zwischen einem Kunden und einem Anbieter von Nachbarschaftsdiensten oder Kinderbetreuungen angestrebt wird.

\section{Design Challenge: Service-Ökosystem}

Die Projekte der Förderlinie „Personennahe Dienstleistungen" haben sich frühzeitig mit der Entwicklung geeigneter Geschäftsmodelle auseinandergesetzt. In einem ersten Workshop zu Service-Ökosystemen beleuchtete ein Impuls- vortrag die Herausforderung mehrerer Projekte, mit einer digitalen Vermittlung Umsatz zu generieren. Hier gilt der Merksatz: Viele Köche gestalten den Brei. Suchen Sie Mitstreiter, nicht nur Kunden.

Die Methode Design ES (Ecosystem) wertorientiert (vgl. Abb. 5) visualisiert mithilfe von Symbolkarten für die Akteure, ihre (digitalen) Services und Kompetenzen zunächst die Dreiecksbeziehung eines zweiseitigen Marktes. Akteur D (Dienstleister) bietet z.B. Nachhilfe (Service) an, die Vermittlungsplattform $\mathrm{V}$ vermittelt (Matching) an einen Nachhilfekunden K und dieser vergütet D (mit der Kompetenz Geld). Verlangt V hierfür Provision, wird K bei erneutem Nachhilfebedarf voraussichtlich direkt D anfragen und $\mathrm{V}$ übergehen. Es stellt sich somit die Frage, wie $\mathrm{V}$ nachhaltig Umsatz generieren kann. Eine weitere Herausforderung für $\mathrm{V}$ besteht darin, überhaupt ausreichend Reichweite zu schaffen, um von D oder K im Netz gefunden zu werden. Mithilfe der Kartensets für Patterns im Bereich von (digitalen) Services (vgl. die vorherigen 
Design Challenges) sowie derjenigen zu potenziellen Akteuren (Entwicklungslinie Integration) und notwendigen oder besonderen Kompetenzen (z. B. Reichweite, Wissen über den Markt), kann nun das Team die Konstellation aus $\mathrm{D}, \mathrm{K}$ und $\mathrm{V}$ schrittweise erweitern und diskutieren. So schlägt beispielsweise die Kinderbetreuungsplattform von KUSTOMA vor, als weitere Dienstleistung Betreuer:innen verschiedene Lernspiele zur Verfügung zu stellen, die potenziell den Wert der Betreuung für die Eltern erhöhen, sodass diese bereit sind, KUSTOMA hierfür zu bezahlen. Oder die Plattform bindet den Akteur Unternehmen in das Service-Ökosystem ein. Dessen Wert eingebunden zu werden liegt darin, seinen Mitarbeitern bei akutem Bedarf eine Kinderbetreuung zu ermöglichen. Dies verbessert die Lebenssituation des einzelnen Mitarbeiters, der beruhigt seiner beruflichen Tätigkeit nachgehen kann, und bietet zudem dem Unternehmen den Wert einen möglichen Personalausfall zu vermeiden. Gleichzeitig trägt das Unternehmen potenziell die Kompetenz Reichweite bei, sodass die Plattform jeweils gleichzeitig für viele Eltern bekannt wird.

Weitere Kartensets zu Patterns aus der Entwicklungslinie digitales Design ergänzen Design ES wertorientiert hinsichtlich der potenziell erfolgreichen Gestaltung einer digitalen Plattform in verschiedenen Entwicklungsstufen (Patterns zu Plattform Life). Sie bieten Anregungen beispielsweise dahingehend, über die Skalierbarkeit digitaler Leistungen oder über Netzeffekte auf digitalen Märkten nachzudenken. Hier zeigen Erkenntnisse aus einer aktuellen Studie des BeDien-Projekts auf, wie eine digitale Plattform das Wohlbefinden ihrer Nutzer und damit deren Verbundenheit steigern kann [22].

Im Anschluss an den ersten Workshop zu Service-Ökosystemen entwickelten eine Reihe von Projekten wesentliche Aktivitäten, so nutzte beispielsweise $A R B A Y$ die Methode Design ES wertorientiert für die Zusammenarbeit im Projekt. Von den 8 beteiligten Projekten forderten 5 in der Zwischenzeit weitere Workshops ein. Mit einem davon, Athene 4.0, erarbeitete das BeDien-Team Methoden für den Zeitpunkt, in dem ein Projekt ein erstes funktionierendes Service-Ökosystem konzipiert hat. Beispielsweise stellt eine Wert-Kompetenzen-Matrix für alle Akteure zusammen, ob sie jeweils Kompetenzen in das ServiceÖkosystem einbringen und dafür auch selber wertvolle Services anderer Akteure erhalten. Denn ein Akteur, der für sich keinen Nutzen aus einem Service-Ökosystem ziehen kann, wird diesem nicht nachhaltig erhalten bleiben. Anschließend kann jeder einzelne Akteur mithilfe eines WertKompetenzen-Checks detailliert prüfen, ob seine eigenen und die im Ökosystem vorhandenen fremden Kompetenzen zum einen seinen Value in Use sicherstellen und zum anderen zu Services führen, die mit diesem vereinbar sind. Das Projekt $M Y O W$, beispielsweise, konnte damit die Werte für alle Partner schärfen und stellte fest, dass es im derzeitigen Ökosystem nicht im Sinne des Value in Use eines Designers für Wearables ist, seine Pläne auf der digitalen Plattform offen zu legen. Finden sich, wie hier, Lücken oder kritische Aspekte im Service-Ökosystem, werden schrittweise erneut Methoden für den Value in Use, den Value in Interaction oder direkt Design ES wertorientiert angewandt.

\section{Fazit}

Kundenorientiert zu handeln, ist schon seit vielen Jahren die Grundlage wirtschaftlichen Handelns. Auf digitalen Märkten jedoch geht die Orientierung an Kunden weit darüber hinaus, ein Produkt nach ihren Präferenzen zu gestalten. Stattdessen begleiten digitale personennahe Dienstleistungen jeden Menschen in engen Beziehungen zwischen Anbieter und Nachfrager durch sein Leben. Mit der Service Logik wird in einer neuen Perspektive auf Märkte beschrieben, wie betriebliche Angebote daran ausgerichtet sein sollten, die Lebenssituation von Menschen zu verbessern. Die in diesem Beitrag dargestellte Service Canvas mit ihren Designmethoden geht über die Erklärung der neuen Marktgegebenheiten aus der Service Logik hinaus und bietet aktive Hilfe in der Gestaltung (digitaler) personennaher Dienstleistungen. Erste Erfahrungen im Einsatz des neuen Service-Design-Methodensets im Rahmen des Metaprojekts BeDien der BMBF-Förderlinie „Personennahe Dienstleistungen“ zeigen, dass in entsprechenden Workshops neue Ideen für besser am Kunden ausgerichtete, aber auch nachhaltig effektive Dienstleistungen und deren Service-Ökosystem entstehen.

Damit ist dem wirtschaftlichen Handeln nach der neuen, direkt am Wohlergehen der Menschen ausgerichteten und in der digitalen Transformation Erfolg versprechenden Prämisse Service for Good der Weg geebnet. Natürlich fällt es nicht jedem industriellen Unternehmen, beispielsweise aus dem B2B-Bereich, so leicht, sein Produkt als personennahe Dienstleistung zu gestalten, wie den Projekten aus der personennahen Dienstleistung. Das Gedankenexperiment jedoch, auch einen betrieblichen Kunden als Akteur zu sehen, der seinen Mitarbeitern oder einem Endkunden letztlich einen individuellen Value in Use bieten sollte, lohnt sich - wie es der ständige Erkenntnisfortschritt der Service Logik in der wissenschaftlichen und auch praxisnahen Diskussion zeigt.

Die Community Service for Good sieht es hier als ihre Aufgabe für alle Unternehmen und Organisationen im deutschsprachigen Raum, anschlussfähig und mit weiteren, ergänzten und angepassten Methoden wertvoll zu sein. Dazu gehört es beispielsweise auch, eine neue Servicekultur zu vermitteln, die Dienstleistungen eben nicht ,zu einem Produkt“, sondern „für einen Menschen“ entwickelt und zudem 
im Sinne der Interaktionsarbeit auch den Mitarbeiter eines Unternehmens als für sich wertschaffenden, aktiven Akteur anerkennt [24].

Dabei ist die Initiative Service for Good selber auch eine personennahe Dienstleistung, die Unternehmen und Organisationen einen Value in Use verspricht, diesen durch Interaktionen während Workshops und im digitalen Raum (www.service-for-good.de) vermittelt und auf ein ServiceÖkosystem verschiedener Akteure aufbaut. Wir freuen uns, unter den Lesern dieses Beitrags weitere Community-Mitglieder zu finden.

Förderung Dieser Beitrag ist Teil des vom Bundesministerium für Bildung und Forschung geförderten Verbundprojekts „BeDien - Begleitforschung Personennahe Dienstleistungen“ (Förderkennzeichen: 02K17A080-81).

Funding Open Access funding enabled and organized by Projekt DEAL.

Open Access Dieser Artikel wird unter der Creative Commons Namensnennung 4.0 International Lizenz veröffentlicht, welche die Nutzung, Vervielfältigung, Bearbeitung, Verbreitung und Wiedergabe in jeglichem Medium und Format erlaubt, sofern Sie den/die ursprünglichen Autor(en) und die Quelle ordnungsgemäß nennen, einen Link zur Creative Commons Lizenz beifügen und angeben, ob Änderungen vorgenommen wurden.

Die in diesem Artikel enthaltenen Bilder und sonstiges Drittmaterial unterliegen ebenfalls der genannten Creative Commons Lizenz, sofern sich aus der Abbildungslegende nichts anderes ergibt. Sofern das betreffende Material nicht unter der genannten Creative Commons Lizenz steht und die betreffende Handlung nicht nach gesetzlichen Vorschriften erlaubt ist, ist für die oben aufgeführten Weiterverwendungen des Materials die Einwilligung des jeweiligen Rechteinhabers einzuholen.

Weitere Details zur Lizenz entnehmen Sie bitte der Lizenzinformation auf http://creativecommons.org/licenses/by/4.0/deed.de.

\section{Literatur}

1. Zuboff S (2010) Creating value in the age of distributed capitalism. Mckinsey Q 12(1):1-12

2. Robra-Bissantz S, Lux AM, Lattemann C, Ziegler C (2021) Leiden Sie unter Symptomen der digitalen Transformation? Personennahe Dienstleistungen helfen. In: Schulz (Hrsg) Industrie 4.0 - Lean Services als Grundlage des Dienstleistungsgeschäfts. Springer Gabler, Wiesbaden, S 21-37

3. Schreiner N, Kenning P (2018) Teilen statt Besitzen: Disruption im Rahmen der Sharing Economy. In: Keuper F, Schomann M, Sikora L, Wassef R (Hrsg) Disruption und Transformation Management. Springer Gabler, Wiesbaden

4. Ostrom AL, Bitner MJ, Brown SW, Burkhard KA, Goul M, SmithDaniels V, Rabinovich E (2010) Moving forward and making a dif- ference: research priorities for the science of service. J Serv Res 13(1):4-36

5. Grönroos C (2006) Adopting a service logic for marketing. Mark Theory 6:317-333

6. Grönroos C (2011) Value co-creation in service logic: a critical analysis. Mark Theory 11(3):279-301

7. Vargo SL, Lusch RF (2004) Evolving to a new dominant logic for marketing. J Mark 68(1):1-17

8. Robra-Bissantz S, Lattemann C (2017) 7 rules of attraction. HMD 54(5):639-658

9. Vargo SL, Lusch RF (2008) Service-dominant logic—continuing the evolution. J Acad Mark Sci 36(1):1-10

10. Grönroos C (2008) Service logic revisited: who creates value? And who co-creates? Eur Bus Rev 20(4):298-314

11. Geiger M, Robra-Bissantz S, Meyer M (2020) Wie aus digitalen Services Wert entsteht: Interaktionen richtig gestalten. HMD 57(2):385-398

12. Vargo SL, Lusch RF (2016) Institutions and axioms: an extension and update of service-dominant logic. J of the Acad Mark Sci 44:5-23

13. Rust RT, Huang M-H (2014) The service revolution and the transformation of marketing science. Mark Sci 33(2):206-221

14. Fischer S, Lux AM, Guerrero R, Ahmad R, Lohrenz L, Lattemann C (2020) Digitalisierung als Grundlage wertvoller $\mathrm{Zu}$ sammenarbeit - Die Gestaltung von Service-Ökosystemen in den personennahen Dienstleistungen. HMD 57(1):655-668

15. Greiner B, Teubner T, Weinhardt C (2018) Grundfragen der Plattformökonomie - Wie man Vertrauen designt. Nomos,

16. BeDien.org (2019) Personennahe Dienstleistungen gemeinsam gestalten. https://www.bedien.org. Zugegriffen: 22.07.2021

17. Robra-Bissantz S, Lattemann C, Guerrero R, Lux AM, Redlich B, Fischer S (2020) Der Mensch als Teil der Innovation Eine „Service Canvas“ als anwendungsorientierter Bezugsrahmen. In: Bruhn M, Hadwich K (Hrsg) Automatisierung und Personalisierung von Dienstleistungen. Springer Gabler, Wiesbaden

18. BeDien.org: Susns Service Storys Teil 6: Die Wahrheit zum Value in Interaction, BeDien.org (2019)

19. Blunk O, Brown G, Prilla M (2020) Möglichkeiten der Digitalisierung zur Beratung von hochkonfigurierbaren und hochindividualisierbaren Gütern. HMD 57:669-683

20. Leiner P, Ruppert E, Eiletz M et al (2020) Do-It-Yourself-Plattformen für individuelle Wearables - Konzeption einer Maker-Plattform und Potenzialanalyse. HMD 57:744-753

21. Mehrwald P, Heymann F (2020) Die Digitalisierung von Personenbezogenen Dienstleistungen durch Online-Plattformen: Woher kommt die geringe Nutzung? HMD 57:767-782

22. Lohrenz L, Michalke S, Robra-Bissantz S, Lattemann C (2021) Mechanismen zur Gestaltung erfolgreicher digitaler Plattformen. Wirtschaftsinform Manag 13:132-142

23. Zukunftsinstitut (2021) Megatrend Konnektivität. zukunftsinstitut.de/dossier/megatrend-konnektivitaet. Zugegriffen: 22.07.2021

24. Robra-Bissantz S, Lattemann Ch (2021) Do you practice what you preach? Aktionsfelder und praktische Schritte für den unternehmerischen Wandel zur Service-Kultur. Leitartikel Service Today $02 / 2021$ 